\title{
Lean rats gained more body weight than obese ones from a high-fibre diet
}

\author{
Shaoting Li†, Cheng Zhang†, Yingyi Gu, Long Chen, Shiyi Ou, Yong Wang and Xichun Peng* \\ Department of Food Science and Engineering, Jinan University, Guangzhou 510632, China
}

(Submitted 17 March 2015 - Final revision received 26 June 2015 - Accepted 30 June 2015 - First published online 28 August 2015)

\section{Abstract}

There is controversy over previous findings that a high ratio of Firmicutes to Bacteriodetes helps obese animals harvest energy from the diet. To further investigate the relationship between microbial composition and energy harvest, microbial adaptation to diet and time should be considered. In this study, lean and obese rats were successfully induced with low-fat and high-fat diets. An 8-week high soyabean fibre (HSF)-containing diet was then fed to investigate the interaction between the diet and the rats' gut microbiota, as well as their influence on rats' growth. Rats' body weight (BW) was recorded weekly; their plasma lipids and their gut microbiota at week 11, 15 and 19 were analysed. After the consumption of the HSF diet, BW of lean rats increased significantly $(P<0.05)$, but no significant alteration in BW was found in obese rats. The average content of plasma cholesterol was lowered and that of TAG was upgraded in both the groups when fed the HSF diet. There was no significant difference observed at each period between lean and obese rats. In the group of lean rats, the diversity of gut microbiota was elevated strongly $(P<0 \cdot 01)$, and bacteria from phylum Firmicutes and Bacteroidetes were both increased largely $(P<0 \cdot 01)$; however, the bacterial diversity and composition in obese rats were less altered after the HSF diet control. In conclusion, the increased Firmicutes and Bacteriodetes might relate to lean rats' higher BW gain; 'obese microbiota' could not help the hosts harvest more energy from the HSF diet.

Key words: Obesity: Soyabean fibre: Energy harvest: Gut microbiota: Bacteroidetes: Firmicutes

People's weight and body composition are likely determined by interactions between their genetic makeup and social, cultural, behavioural and environmental factors. There is equilibrium between the amount of energy obtained from food and the amount expended through resting metabolism, thermic effect of food, physical activity and loss via faeces and urine. People's increased intake of energy-dense foods surely contribute to the high prevalence of obesity; however, as the internal regulating system of energy balance is far more complex than that we have imagined, the interaction between diet and obesity should be considered in a larger context ${ }^{(1,2)}$.

Recent evidence suggests that gut microbiota can affect nutrient acquisition and energy regulation; moreover, scientists have also discovered that obese and lean people have different gut microbiota $^{(3)}$. Changes in gut microbial ecology can cause alteration in the host's efficiency of energy harvest from the diet, leading to significant changes in body weight (BW) and energy balance ${ }^{(4)}$. Gut microbiota may play an important role in regulating weight, as it has been recently reported that gut bacterial composition is partly responsible for the development of obesity in some people $\mathrm{e}^{(3)}$. Turnbaugh et al. ${ }^{(5)}$ believed that the alteration in gut microbial composition is possibly associated with the onset of obesity, and they also indicated that a high ratio of Firmicutes in an obese individual's gut can help the host harvest more energy from food. Furthermore, the gut microbiota may influence host physiology through several mechanisms - for example, increasing energy harvest from the diet, regulating hormone production in the mucosal lining and increasing the inflammatory tone ${ }^{(6-9)}$.

In our previous study, the energy harvesting abilities of obese and lean rats fed a high-fructo-oligosaccharide diet had been compared. It was discovered that lean rats obtained more energy from the diet and grew faster ${ }^{(10)}$, which has not been reported so far in the literature. In order to validate our previous findings, an insoluble dietary fibre - soyabean fibre - was used to carry out some further studies. Soyabean fibre was selected because of its popular application in the food industry ${ }^{(11)}$ and due to the fact that it is a complex dietary fibre that contains cellulose, pectin and xylan, etc. ${ }^{(12)}$, which can induce the growth of a variety of bacteria. We intended to investigate whether lean rats will still be more capable of energy harvest when ingesting a high soyabean fibre (HSF)-containing diet, and how their gut microbiota will respond to this switch of diet.

\section{Methods}

\section{Animal, diets and sample preparation}

Twenty male Sprague-Dawley rats (5-6 weeks old) (Guangdong Medical Laboratory Animal Center) were housed in a temperaturecontrolled room $\left(23\left(\mathrm{sD} 2^{\circ} \mathrm{C}\right)\right)$ with $12 \mathrm{~h}$-light-12 h-dark cycles.

Abbreviations: BW, body weight; HSF, high soyabean fibre.

* Corresponding author: X. Peng, email tpxchun@jnu.edu.cn

$\dagger$ These authors contributed equally to the present work. 
All the rats had free access to standard chow diet and water. The animal feeding experiment was conducted as descripted in the timeline figure (Fig. 1). After $7 \mathrm{~d}$ adaption, animals were randomly assigned to two experimental groups (eight rats per group): (a) SL group, in which rats were fed the low-fat feed for 10 weeks and then the HSF feed for 8 weeks; and (b) SO group, in which rats were fed high-fat feed for 10 weeks and then the HSF feed for 8 weeks. The feeds were formulated according to AIN-93 diet and Research Diets (D12450B for low-fat feed and D12492 for high-fat feed) with modification ${ }^{(13-15)}$ (Table 1). Animals were housed in independent ventilated cages with four animals each. BW was recorded weekly. Animal tail blood and fresh faecal specimens were individually collected at the end of week 11, 15 and 19. Blood samples and faecal specimens were packaged separately and frozen directly after collection. The blood samples and faecal specimens were stored at $-80^{\circ} \mathrm{C}$. All the rats were killed by decapitation at week 19 . The present study was conducted according to the Guangdong Experimental Animal Guidelines, and all the animal experiments were approved by the Research Animal Administration Center at Jinan University.

\section{Plasma lipid}

After being thawed, blood samples were centrifuged at $2000 \mathrm{rpm}$ for $20 \mathrm{~min}$ and plasma samples were individually collected. Total plasma cholesterol ( $\mathrm{mmol} / \mathrm{l})$ and TAG $(\mathrm{mmol} / \mathrm{l})$ concentrations were assayed as previously described (Cholesterol EZ, Triglyceride EZ) $^{(16)}$.

\section{Faecal bacterial DNA extraction}

The faecal bacterial DNA from each sample was extracted using TIANamp Stool DNA kit (Tiangen) according to the manufacturer's instructions. The total DNA samples were characterised with $1 \%$ agarose gel electrophoresis for integrity and size. The extracted DNA samples were stored at $-80^{\circ} \mathrm{C}$ before being used as templates for 16S rDNA analysis.

\section{5 rDNA gene $P C R$ amplification and sequencing}

The primers F515 (59-CACGGTCGKCGGCGCCATT-39) and R806 (59-GGACTACHVGGGTWTCTAAT-39) ${ }^{(17)}$ were used to amplify

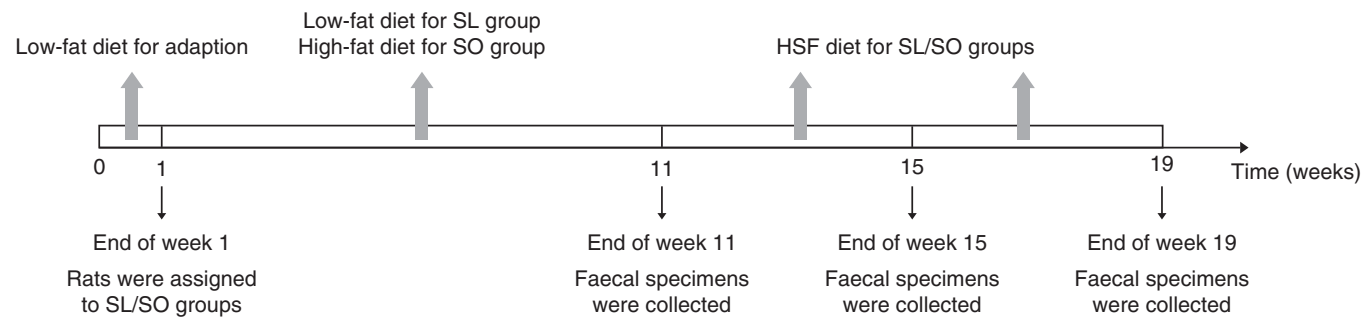

Fig. 1. Timeline figure of the feeding experiment. Week 1 was the adaptation period for the rats. The SL/SO groups of rats were fed low-fat/high-fat diet from the end of week 1 to the end of week 11. The SL group and the SO group of rats were all fed high soyabean fibre (HSF) diet from the end of week 11 to the end of week 19 . The faecal specimens were collected at the end of week 11, 15 and 19. SL, group of lean rats; SO, group of obese rats.

Table 1. Formulae of the low-fat feed, high-fat feed and high soyabean fibre (HSF) feed

\begin{tabular}{|c|c|c|c|c|c|c|c|c|c|}
\hline & \multicolumn{3}{|c|}{ Low-fat feed* } & \multicolumn{3}{|c|}{ High-fat feed $†$} & \multicolumn{3}{|c|}{ HSF feed* } \\
\hline & $g \%$ & Energy \% & & $g \%$ & Energy \% & & $g \%$ & Energy \% & \\
\hline Protein & $19 \cdot 4$ & $20 \cdot 0$ & & $26 \cdot 6$ & $20 \cdot 0$ & & $19 \cdot 4$ & $22 \cdot 2$ & \\
\hline Carbohydrate & 67.9 & $70 \cdot 0$ & & $26 \cdot 8$ & $20 \cdot 1$ & & $58 \cdot 3$ & $66 \cdot 7$ & \\
\hline Fat & $4 \cdot 3$ & $10 \cdot 0$ & & $35 \cdot 3$ & 59.9 & & $4 \cdot 3$ & $11 \cdot 1$ & \\
\hline Energy (kJ/g) & & $16 \cdot 2$ & & & $22 \cdot 2$ & & & 14.6 & \\
\hline \multirow[t]{2}{*}{ Energy $(\mathrm{kcal} / \mathrm{g}$ ) } & & 3.88 & & & $5 \cdot 31$ & & & 3.50 & \\
\hline & g & kcal & $\mathrm{kJ}$ & g & kcal & kJ & g & kcal & kJ \\
\hline \multicolumn{10}{|l|}{ Ingredient } \\
\hline Casein & 200 & 800 & $3347 \cdot 2$ & 200 & 800 & $3347 \cdot 2$ & 200 & 800 & $3347 \cdot 2$ \\
\hline L-Cystine & 3 & 12 & $50 \cdot 2$ & 3 & 12 & $50 \cdot 2$ & 3 & 12 & $50 \cdot 2$ \\
\hline Maize starch & 315 & 1260 & $5271 \cdot 8$ & 0 & 0 & 0 & 315 & 1260 & 5271.8 \\
\hline Maltodextrin & 35 & 140 & $585 \cdot 8$ & 125 & 500 & 2092 & 35 & 140 & 585.8 \\
\hline Sucrose & 350 & 1400 & $5857 \cdot 6$ & 68.8 & $275 \cdot 2$ & 1151.4 & 250 & 1000 & 4184 \\
\hline Soyabean fibreł & 50 & 0 & 0 & 50 & 0 & 0 & 150 & 0 & 0 \\
\hline Soyabean oil & 25 & 225 & 941.4 & 25 & 225 & 941.4 & 25 & 225 & 941.4 \\
\hline Lard & 20 & 180 & $753 \cdot 1$ & 245 & 2205 & $9225 \cdot 7$ & 20 & 180 & 753.1 \\
\hline Mineral mix AIN-93 & 35 & 0 & 0 & 35 & 0 & 0 & 35 & 0 & 0 \\
\hline Vitamin AIN-93 & 10 & 40 & $167 \cdot 3$ & 10 & 40 & $167 \cdot 3$ & 10 & 40 & $167 \cdot 3$ \\
\hline Choline bitartrate & 2.5 & 0 & 0 & 2.5 & 0 & 0 & 2.5 & 0 & 0 \\
\hline Total & $1045 \cdot 5$ & 4057 & 16974.5 & $764 \cdot 3$ & $4057 \cdot 2$ & 16975.3 & $1045 \cdot 5$ & 3657 & $15300 \cdot 9$ \\
\hline
\end{tabular}

* The feeds were formulated according to AIN-93 diet and D12450B of Research Diets with modification.

† The feeds were formulated according to AIN-93 diet and D12492 of Research Diets with modification.

$\ddagger$ The dietary fibre used in these feeds was soyabean fibre. 
the $\mathrm{V}_{4}$ domain ${ }^{(18)}$ of bacterial $16 \mathrm{~S}$ rDNA. The length of the amplified $\mathrm{V}_{4}$ region was $241 \mathrm{bp}$. PCR reactions contained 5-100 ng DNA template, $1 \times$ GoTaq Green Master Mix (Promega), $1 \mathrm{~mm} \mathrm{MgCl} 2$ and 2 pmol of each primer. Reaction conditions were as follows: an initial $94^{\circ} \mathrm{C}$ for $3 \mathrm{~min}$, followed by thirty-five cycles of $94^{\circ} \mathrm{C}$ for $45 \mathrm{~s}$, $50^{\circ} \mathrm{C}$ for $60 \mathrm{~s}$ and $72^{\circ} \mathrm{C}$ for $90 \mathrm{~s}$ and a final extension of $72^{\circ} \mathrm{C}$ for $10 \mathrm{~min}$. All the samples were amplified in triplicate and combined before purification. Amplicons were purified using the Qiaquick 96 kit (Qiagen), quantified using PicoGreen dsDNA reagent (Invitrogen) and gel-purified using the Qiaquick gel extraction kit (Qiagen), all according to the manufacturers' instructions. Purified libraries were sequenced on the Illumina GAIIx platform.

\section{5 rDNA gene analysis}

Raw Illumina fastq files were de-multiplexed, quality-filtered and analysed using Quantitative Insights Into Microbial Ecology (QIIME) ${ }^{(19)}$. Sequences that were shorter than $55 \mathrm{bp}$, contained primer mismatches, ambiguous bases or uncorrectable barcodes were removed; 16S rDNA gene sequences were assigned to operational taxonomic unit using UCLUST with a threshold of $97 \%$ pair-wise identity ${ }^{(20)}$, and then classified taxonomically using the Ribosomal Database Project (RDP) classifier $2 \cdot 0 \cdot 1^{(21)}$.

Alpha diversity estimates were calculated with Shannon value. Principal coordinates analysis (PCA) was performed to present differences between the gut microbial communities of the two groups. These analyses were conducted by Gene Denovo Co.

\section{Statistical analysis}

Results were expressed as mean values and standard deviations. The statistical analysis was performed with SPSS $17 \cdot 0$ software

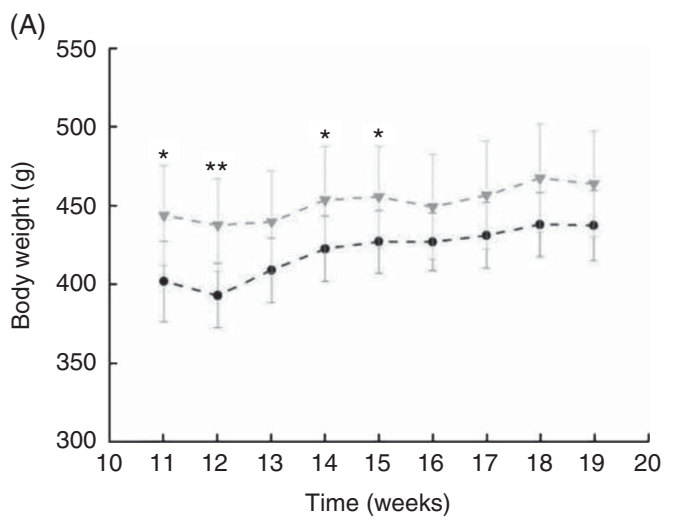

(SPSS Inc.). T-tests were conducted to compare the phenotypes of the lean and obese rats, and all statistical tests were two-tailed. Statistical significance was set at a $P<0.05$.

\section{Results}

\section{Feed consumption and growth of animals}

The models of lean and obese rats were separately induced by 10 weeks' low-fat and high-fat diet. At week 11, the average weight of SO rats was about 443.30 (SD 31.96) g, higher than 401.30 (sD 25.75) g of SL rats, with a significant difference $(P<0.05)$ (Table 2$)$, indicating that the models of lean and obese rats were successfully built. When the feed was switched to the HSF diet, all the rats experienced a period of adaptation (1 week); during week 12, the BW gains of SL and SO rats were both negative. The BW of all rats increased slowly from then on. From week 16 onwards, there were no significant differences between the BW of SL and SO groups (Fig. 2(A)). In addition, the BW gain of lean rats was significantly higher than that of obese rats at week 13 and $16(P<0 \cdot 01)$ (Fig. 2(B)). In the end, the total BW gain of SL rats was 35.70 (SD 8.33$) \mathrm{g}$, significantly higher than that of SO rats $(20 \cdot 29$ (SD 7.21) g) $(P<0.01)$; the BW of SL rats reached 437.00 (SD 22.39) g, while that of SO rats reached 463.59 (SD 33.74) g, but no significant difference between them was observed (Table 2).

\section{Changes of plasma lipids}

No significant difference was observed in the contents of plasma cholesterol and TAG between obese and lean rats before they consumed the HSF diet $(P>0.05)$ (Fig. 3). The
(B)

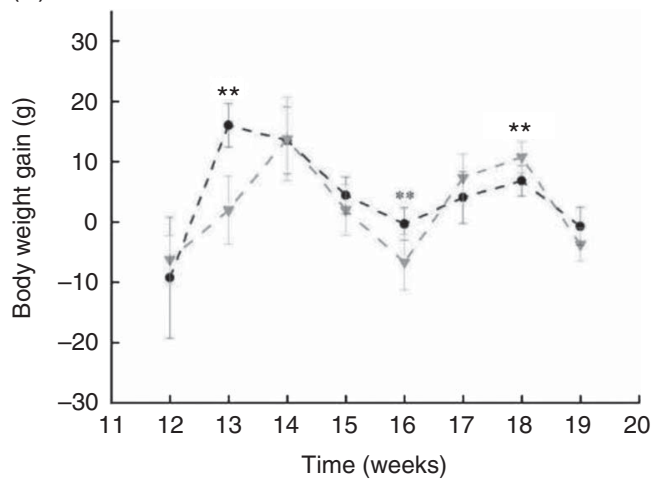

Fig. 2. (A) Body weight (BW) of lean and obese rats when ingesting the high soyabean fibre (HSF) diet. All the rats were fed the HSF diet. (B) BW gain of lean (SL) and obese (SO) rats when ingesting the HSF diet. All the rats were fed the HSF diet. The data at week 12 show the total weight of feed intake from week 11 to 12 . Values are means, with standard deviations represented by vertical bars. Significant difference between rats of the $\mathrm{SL}$ and $\mathrm{SO}$ groups: ${ }^{\star} P<0.05,{ }^{\star *} P<0.01 .-\bullet-, \mathrm{SL} ;-{ }^{*}-, \mathrm{SO}$.

Table 2. Body weight (BW) and BW gain of lean and obese rats when ingesting the high soyabean fibre (HSF) diet (Mean values and standard deviations)

\begin{tabular}{|c|c|c|c|c|c|c|}
\hline \multirow[b]{2}{*}{ Group (g; $n$ 8) } & \multicolumn{2}{|c|}{ BW (week 11) } & \multicolumn{2}{|c|}{ BW (week 19) } & \multicolumn{2}{|c|}{ BW gain (from week 11 to 19 ) } \\
\hline & Mean & SD & Mean & $\mathrm{SD}$ & Mean & SD \\
\hline SL & $401 \cdot 30^{\mathrm{a}}$ & $25 \cdot 75$ & $437 \cdot 00^{b}$ & $22 \cdot 39$ & $35 \cdot 70$ & 8.33 \\
\hline SO & $443 \cdot 30^{*}$ & 31.96 & 463.59 & $33 \cdot 74$ & $20 \cdot 29^{*}$ & $7 \cdot 21$ \\
\hline
\end{tabular}

$\mathrm{SL}$, group of lean rats; SO, group of obese rats.

a,b Mean values within a row with unlike superscript letters were significantly different $(P<0.05)$.

* Mean value was significantly different from that of the SL group $(P<0.05)$. 
(A)

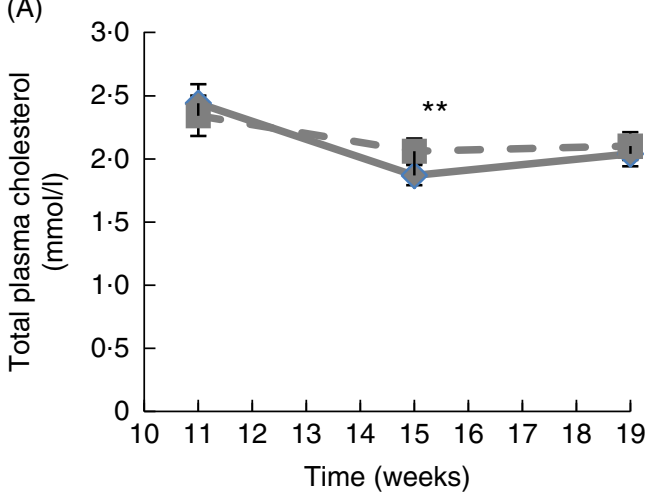

(B)

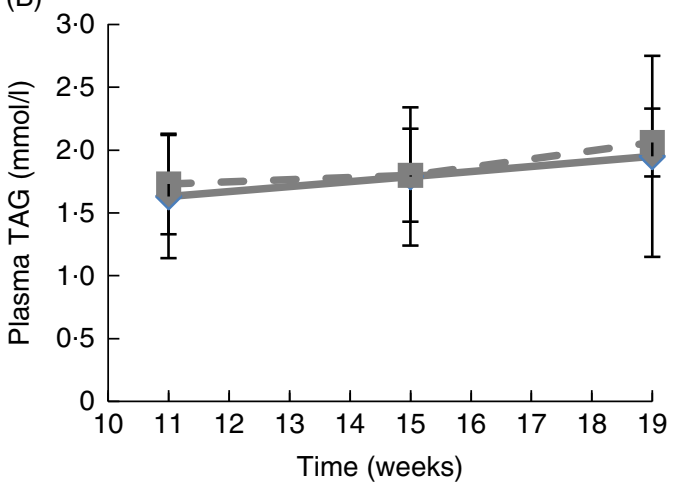

Fig. 3. Plasma lipids in lean and obese rats. (A) Total cholesterol; (B) TAG. ${ }^{* *}$ Significant difference between groups $(P<0.01)$. SL, group of lean rats; SO, group of obese rats. $\_-, \mathrm{SL} ;-\square$, SO.

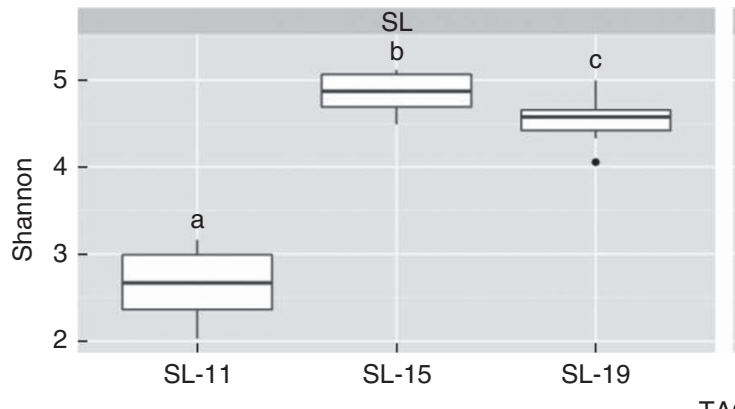

TAG

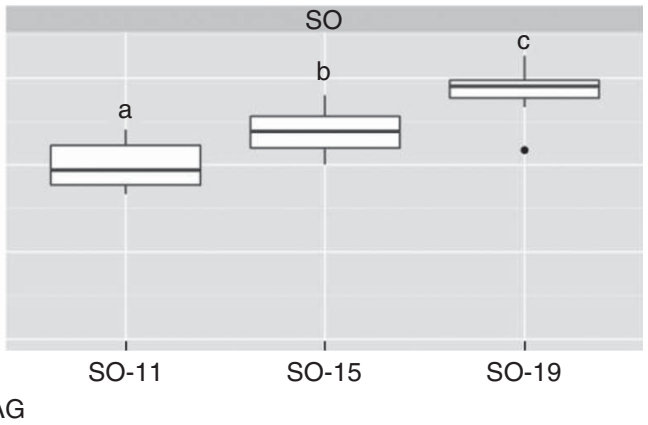

Fig. 4. Shannon values of the gut microbiota in lean and obese rats. Shannon value indicates the alpha diversity of the rats' gut microbiota. The higher the Shannon value, the higher the diversity of microbiota. Shannon values were presented with the box plots. The characters 11,15 and 19, respectively, represent week 11 (SL-11, $N$ 8; SO-11, $N$ 8), week 15 (SL-15, $N 7$; SO-15, $N 7$ ) and week 19 (SL-19, $N 7$; SO-19, $N$ 8). ${ }^{\text {a,b,c }}$ Values with unlike letters were significantly different $(P<0.05)$. $\mathrm{SL}$, group of lean rats; SO, group of obese rats.

average content of plasma cholesterol decreased in both SL and SO groups when fed the HSF diet, but it was significantly higher in the SO group at week $15(P<0 \cdot 01)$, and the value became similar in both groups at week 19. Due to the HSF diet supplementation for 8 weeks, the average contents of TAG were upgraded in both groups. There was no significant difference observed at each period between the SL and SO groups.

\section{Variation of faecal microbial communities in lean and obese rats}

According to the Shannon values obtained (Fig. 4), the intestinal microbial diversity in both SL and SO rats increased significantly $(P<0.01$ and $<0.05)$ after the consumption of the HSF diet; the alpha diversity of SL rats became significantly higher than that of SO rats at week $15(P<0 \cdot 01)$. From week 15 to 19 , the Shannon value of SL rats decreased slightly $(P<0.05)$ but that of SO rats increased $(P<0.05)$; there was no significant difference between these two groups at the end of week 19. The figure of PCA demonstrated the similarity between samples (Fig. 5). At week 11, the clustered sample points of SL rats distinctly separated from those of SO-I rats, suggesting that the gut microbiota of lean and obese rats were significantly different. After the new diet was ingested for 8 weeks, the bacterial communities of all the rats tended to be more similar.
At week 11 (Fig. 6(A)), lean rats had more of Proteobacteria $(P<0.01)$ and lesser of Firmicutes $(P<0.01)$ and Bacteriodetes $(P<0.01)$ compared with obese rats; the relative abundance of Bacteriodetes in lean rats was as low as $0.2 \%$, while that of obese rats was much more considerable (3.93\%). After the consumption of the HSF diet, the abundances of Bacteriodetes in all the groups significantly increased $(P<0 \cdot 01)$, especially the SL group had more Bacteriodetes than the SO group at the end of week 19 (16.69 v. $8.90 \%$, $P<0.01)$. The abundance of Firmicutes increased largely from 25.57 to $65.00 \%(P<0.01)$ in the SL group, but had no significant change in the SO group (from 74.29 to $74.24 \%$ ). However, the ratio of Firmicutes to Bacteriodetes in all the groups declined sharply from 128.48 to $3.89(P<0 \cdot 01)$ in the SL group and slightly from 18.93 to $8.34(P<0.01)$ in the SO group (data not shown).

Fig. 6(B) shows the compositional alternation of gut microbiota at the bacterial family level. From week 11 to 19 , a significant reduction in Desulfovibronaceae was detected $(P<0.01)$ in the SL group, and an increase in several families such as Ruminococcaceae, S24-7, Bacteroidaceae, Lachnospiraceae and Prevotellaceae was observed $(P<0 \cdot 01)$ too. For the SO group, Lactobacillaceae, Lachnospiraceae and Desulfovibrionaceae were found to be decreased $(P<0.05)$, but Ruminococcaceae, Prevotellaceae and Bacteroidaceae were found to be significantly increased $(P<0.05)$. 


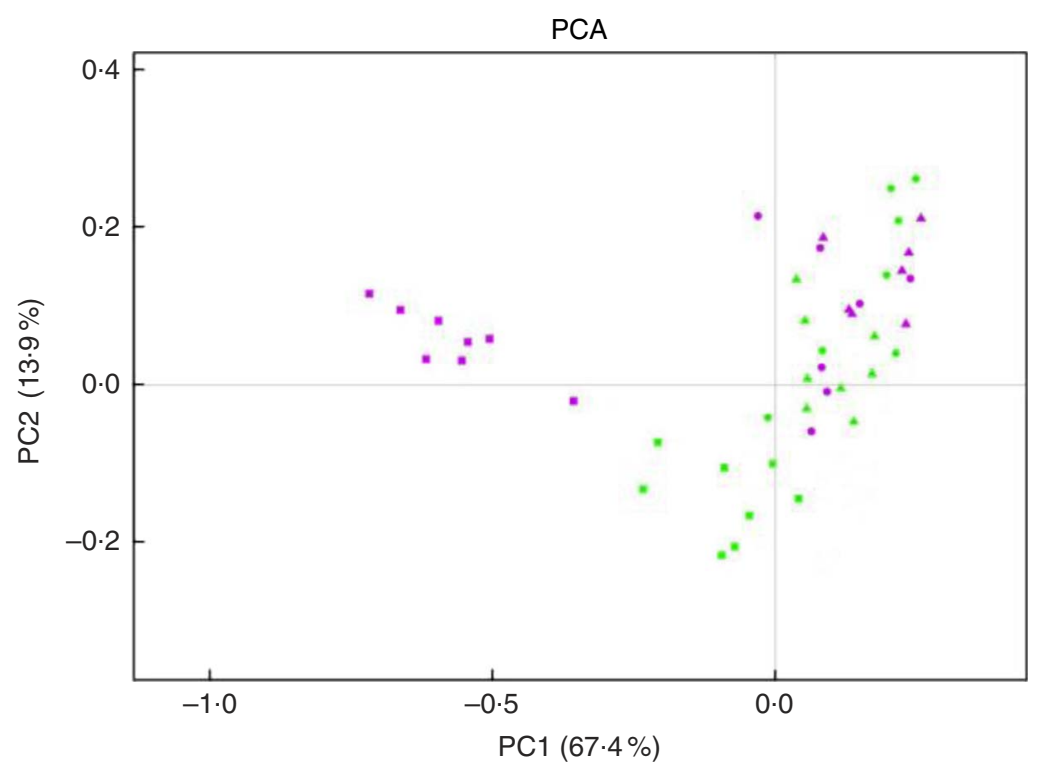

Fig. 5. Principal coordinates analysis (PCA) of the gut microbiota in lean and obese rats. PCA was plotted based on the family level. PCA was plotted to summarise the microbial compositional differences between samples of different groups. Points that are closer represent microbial communities that are more similar. The characters 11, 15 and 19, respectively, represent week 11, 15 and 19. SL, group of lean rats (purple icons); SO, group of obese rats (green icons); week 11 ( $\mathrm{SL}-11$, $N$ 8; ( SO-11, N 8), week 15 (O SL-15, N7; SO-15, N 7), week 19 ( $\triangle \mathrm{SL}-19, N 7 ; \triangle$ SO-19, $N$ 8).

(A)

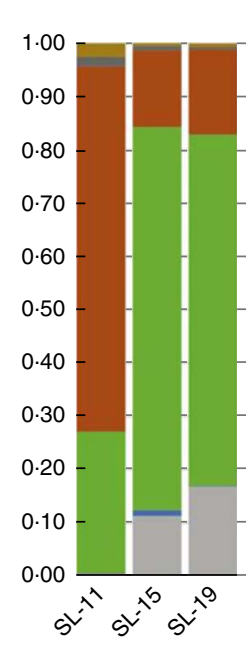

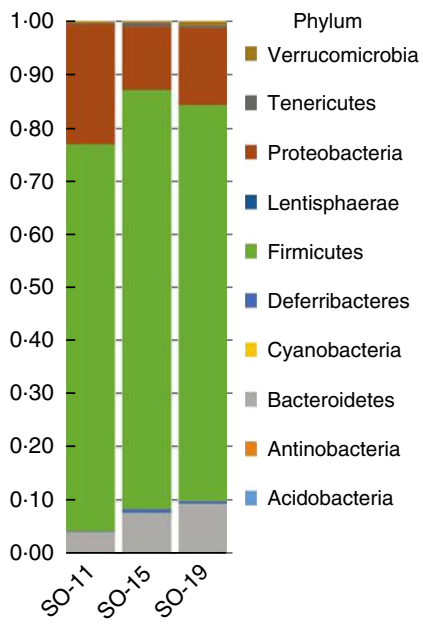

(B)

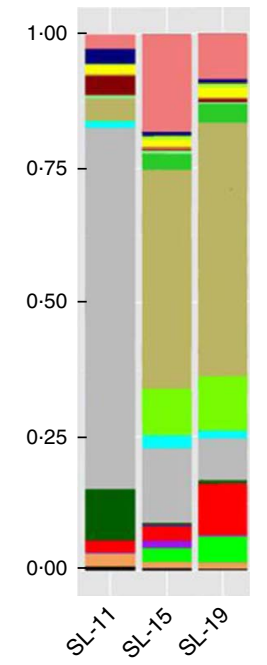

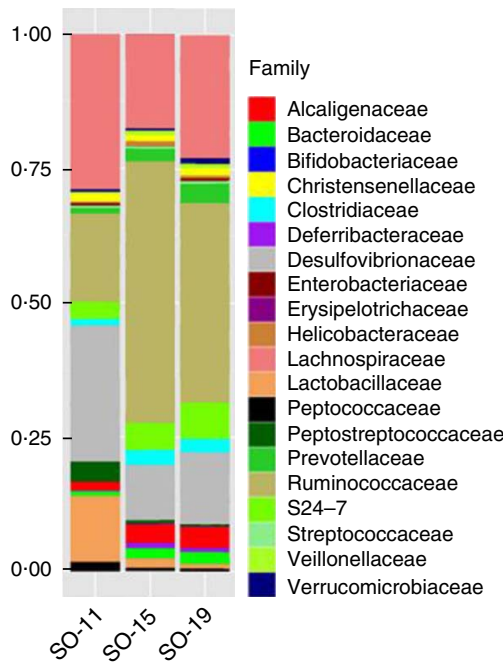

Fig. 6. (A) Relative abundances of the gut microbiota at the phylum level in lean and obese rats. The stack column diagram indicates the bacterial phyla composition of different groups, and different colours represent different kinds of bacterial phyla. (B) Relative abundances of gut microbiota at the family level in lean and obese rats. The stack column diagram indicates the bacterial family composition of different groups, and different colours represent different kinds of bacterial family. The characters 11, 15 and 19, respectively, represent week 11 (SL-11, N 8; SO-11, N 8), week 15 (SL-15, N 7; SO-15, N7) and week 19 (SL-19, N7; SO-19, N 8). $\mathrm{SL}$, group of lean rats; SO, group of obese rats.

\section{Discussion}

In this study, lean and obese rats were individually fed low-fat and high-fat diets, and their gut microbiota was differently shaped. After consumption of the HSF diet, lean rats obtained more BW gain than the obese rats (Table 2). When the rats were fed the HSF diet, the contents of plasma cholesterol was down-regulated and that of TAG was up-regulated in both lean and obese rats. Due to the new diet, lean and obese rats developed a similar composition of gut microbiota, and the gut microbiota of lean rats experienced a more drastic change. As a result, the amounts of Bacteriodetes in lean rats significantly increased and the ratio of Firmicutes to Bacteriodetes largely declined.

Soluble dietary fibres can reduce plasma cholesterol, and insoluble dietary fibres can accelerate intestinal transit, increasing faecal bulk and slowing down the hydrolysis of glucose ${ }^{(22)}$. A high-fibre diet has thought to lower the risk of obesity ${ }^{(22)}$. In this study, soyabean fibre (a mixture of soluble and insoluble fibre) decreased the content of total plasma cholesterol, which is in agreedment with previous reports. However, the content of 
plasma TAG kept increasing along with the growth of rats' BW during the period of ingesting the HSF diet. Tovar et al. ${ }^{(23)}$ reported that a soluble dietary fibre (inulin) can decrease the content of plasma TAG, which was inconsistent with our results. Besides, no relationship was found between plasma lipids and higher BW gain in lean rats.

Previous studies have reported that the high ratio of Firmicutes to Bacteriodetes may assist the obese host harvest more energy from the diet ${ }^{(24-26)}$. However, this opinion has not been confirmed by recent studies; in fact, the opposite results have been reported ${ }^{(10,27)}$. In this study, we also found that the decreased ratio of Firmicutes to Bacteriodetes may relate to rats' increased energy harvest from the HSF diet. As the proportion of soyabean fibre in rats' feed is increased from 5 to $15 \%$, microbes that could secrete glycosyl hydrolases with high activity would be well induced. When the HSF diet was ingested by lean rats, the abundance of Bacteriodetes was found to be largely increased, especially families S24-7, Bacteroidaceae and Prevotellaceae, which belong to the phylum Bacteriodetes, were found to be significantly increased $(P<0 \cdot 01)$ (Fig. 6). In addition, the number of Firmicutes also increased, and families in phylum Firmicutes with the active glycosyl hydrolases were also increased, such as Ruminococcaceae $^{(28)}$ and Lachnospiraceae ${ }^{(29)}$.

In fact, Firmicutes and Bacteriodetes are both capable of degrading polysaccharides. Evidence showed that Bacteroidetes can be regarded as specialists of the degradation of molecules with high molecular mass, such as proteins and carbohydrates ${ }^{(30,31)}$. Bacteroidetes can breakdown a wide variety of indigestible dietary plant polysaccharides (e.g. amylose, cellulose, pectin and xylan) and their predicted glycosyl hydrolases ( $\alpha$-galactosidases, $\beta$-galactosidases, $\alpha$-glucosidases, $\beta$-glucosidases, $\beta$-glucuronidases, $\beta$-fructofuranosidases, $\alpha$-mannosidases, amylases and endo-1, $2-\beta$-xylanases, plus fourteen other activities) exceed that of any other sequenced bacteria ${ }^{(32,33)}$. Therefore, it is understandable that the HSF diet induced large amounts of Bacteriodetes in rats' gut microbiota in this study. Besides, many anaerobic bacteria of phylum Firmicutes from the rumen and human colon are also found capable of degrading polysaccharides, but their gene abundances of encoded glycoside hydrolases are much lesser than polysaccharide-degrading bacteria of phylum Bacteroidetes ${ }^{(34)}$. In this study, Firmicutes were found to be elevated by the HSF diet too, but their contribution to energy harvest was probably not as significant as Bacteroidetes, due to their limited polysaccharide degradation ability. The abundances of Bacteroides (phylum Bacteroidetes), Prevotella (phylum Bacteroidetes), Ruminococcus (phylum Firmicutes) and Blautia (phylum Firmicutes) were found to be significantly increased, and these species could be more capable in the utilisation of the HSF diet.

Murphy et $a l .{ }^{(35)}$ indicated in their study that the relationship between the microbial composition and energy harvest is more complex than that previously considered. Therefore, the microbial adaptation to diet and time was taken into further consideration in this study. One significant finding in this study is that the low-diversity gut microbiota of lean rats was induced to be a relatively high-diversity gut microbiota by the consumption of the HSF diet; on the contrary, the diversity of obese rats' gut microbiota was at a relatively high level before their consumption of the HSF diet. Based on these results, we hypothesised that the increasingly developing bacteria of Bacteroidetes and Firmicutes might be involved in lean rats' higher BW gain from the HSF diet. In addition, the 'obese microbiota' did not help the obese hosts harvest more energy from the HSF diet, as the SO group of rats did not gain more BW than the SL group. The mechanism for the interaction between gut microbiota and energy harvest deserves to be further explored.

\section{Conclusion}

The obese rats did not gain more BW than the lean ones, but no relationship was discovered between plasma lipids and BW gain in rats. The high ratio of Firmicutes to Bacteriodetes in obese rats could not help the hosts gain more BW from the HSF diet; however, lean rats that developed large amounts of Firmicutes and Bacteriodetes obtained higher BW gain. Thus, the increased Firmicutes and Bacteriodetes may relate to lean rats' higher BW gain; 'obese microbiota' could not help the hosts harvest more energy from the HSF diet.

\section{Acknowledgements}

The authors thank Bing $\mathrm{Yu}$ from the Department of Food Science and Engineering, Jinan University, for his contribution to the experiments of this study.

The programme was supported by the funds of the Chinese National Natural Science Funds (no. 31271849) and Nansha District Scientific and Technological Projects (no. 2014GG-05).

S. L. and C. Z. carried out all the experiments and wrote this article. X. P. conceived the study, designed the experiments and drafted the manuscript. Y. G. participated in the statistical analysis. L. C. and S. O. helped revising the manuscript. Y. W. helped designing and instructing the experiments. All the authors read and approved the final version of the manuscript.

There are no conflicts of interest.

\section{References}

1. Hensrud DD \& Klein S (2006) Extreme obesity: a new medical crisis in the United States. Mayo Clin Proc 81, S5-S10.

2. Ogden CL, Yanovski SZ, Carroll MD, et al. (2007) The epidemiology of obesity. Gastroenterology 132, 2087-2102.

3. DiBaise JK, Zhang H, Crowell MD, et al. (2008) Gut microbiota and its possible relationship with obesity. Mayo Clin Proc $\mathbf{8 3}$, 460-469.

4. Flegal KM \& Troiano RP (2000) Changes in the distribution of body mass index of adults and children in the US population. Int J Obes 24, 807-818.

5. Turnbaugh PJ, Hamady M, Yatsunenko T, et al. (2008) A core gut microbiome in obese and lean twins. Nature 457, 480-484.

6. Bäckhed F \& Crawford PA (2010) Coordinated regulation of the metabolome and lipidome at the host-microbial interface. Biochim Biophys Acta, Mol Cell Biol Lipids 1801, 240-245.

7. Cani PD \& Delzenne NM (2009) The role of the gut microbiota in energy metabolism and metabolic disease. Curr Pharm Design 15, 1546-1558.

8. Reinhardt C, Reigstad CS \& Bäckhed F (2009) Intestinal microbiota during infancy and its implications for obesity. J Pediatr Gastroenterol Nutr 48, 249-256.

9. Tilg H, Moschen AR \& Kaser A (2009) Obesity and the microbiota. Gastroenterology 136, 1476-1483. 
10. Li S, Gu Y, Chen L, et al. (2015) Lean rats gained more body weight from a high-fructo-oligosaccharide diet. Food E Funct (Epublication ahead of print).

11. Slavin J (2013) Fiber and prebiotics: mechanisms and health benefits. Nutrients 5, 1417-1435.

12. Madar Z (1983) Effect of brown rice and soyabean dietary fiber on the control of glucose and lipid metabolism in diabetic rats. Am J Clin Nutr 38, 388-393.

13. Gajda AM, Pellizzon MA, Ricci MR, et al. (2007) Diet-induced metabolic syndrome in rodent models. Animal Lab News $\mathbf{7 4}$, 775-793.

14. Reeves PG, Nielsen FH \& Fahey GC Jr (1993) AIN-93 purified diets for laboratory rodents: final report of the American Institute of Nutrition ad hoc writing committee on the reformulation of the AIN-76A rodent diet. J Nutr 123, 1939-1951.

15. Ricci MR \& Ulman EA (2005) Laboratory Animal Diets: a critical part of your in vivo research. Animal Lab News $\mathbf{4}$, $1-5$.

16. Dyson MC, Alloosh M, Vuchetich JP, et al. (2006) Components of metabolic syndrome and coronary artery disease in female ossabaw swine fed excess atherogenic diet. Comparative Med 56, 35- 45 .

17. Caporaso JG, Lauber CL, Walters WA, et al. (2011) Global patterns of $16 \mathrm{~S}$ rRNA diversity at a depth of millions of sequences per sample. Proc Natl Acad Sci USA 108, 4516-4522.

18. Bokulich NA, Joseph CL, Allen G, et al. (2012) Nextgeneration sequencing reveals significant bacterial diversity of botrytized wine. PlOS ONE 7, e36357.

19. Caporaso JG, Kuczynski J, Stombaugh J, et al. (2010) QIIME allows analysis of high-throughput community sequencing data. Nat Methods 7, 335-336.

20. Edgar RC (2010) Search and clustering orders of magnitude faster than BLAST. Bioinformatics 26, 2460-2461.

21. Wang Q, Garrity GM, Tiedje JM, et al. (2007) Naive Bayesian classifier for rapid assignment of rRNA sequences into the new bacterial taxonomy. Appl Environ Microbiol 73, 5261-5267.

22. Queiroz R, Maximiano F, Nunes T, et al. (2008) Avaliação do perfil lipídico, glicêmico, conteúdo de glicogênio hepático e cardíaco em ratos diabéticos suplementados com farinha de casca de maracujá (Passiflora edulis) Rev bras de nutrição clínica (Evaluation of lipid profile, glucose, glycogen contents of liver and heart in diabetic rats supplemented with flour of bark of passionfruit (Passiflora edulis) Rev bras in clinical nutrition). Minas Gerais 23, 173-177.

23. Tovar AR, Caamaño Mdel C, Garcia-Padilla S, et al. (2012) The inclusion of a partial meal replacement with or without inulin to a calorie restricted diet contributes to reach recommended intakes of micronutrients and decrease plasma triglycerides: a randomized clinical trial in obese Mexican women. Nutr J 11, 44.

24. Ley RE, Bäckhed F, Turnbaugh P, et al. (2005) Obesity alters gut microbial ecology. Proc Natl Acad Sci U S A 102, 11070-11075.

25. Mozeš Š, Bujňáková D, Šef̌ćíková Z, et al. (2008) Developmental changes of gut microflora and enzyme activity in rat pups exposed to fat-rich diet. Obesity 16, 2610-2615.

26. Turnbaugh PJ, Bäckhed F, Fulton L, et al. (2008) Diet-induced obesity is linked to marked but reversible alterations in the mouse distal gut microbiome. Cell Host Microbe 3, 213-223.

27. Venema K (2010) Role of gut microbiota in the control of energy and carbohydrate metabolism. Curr Opin Clin Nutr Metab Care 13, 432-438.

28. Cox LM, Cho I, Young SA, et al. (2013) The nonfermentable dietary fiber hydroxypropyl methylcellulose modulates intestinal microbiota. FASEB J 27, 692-702.

29. Dougal K, de la Fuente G, Harris PA, et al. (2013) Identification of a core bacterial community within the large intestine of the horse. PlOS ONE 8, e77660.

30. Dodd D, Mackie RI \& Cann IK (2011) Xylan degradation, a metabolic property shared by rumen and human colonic Bacteroidetes. Mol Microbiol 79, 292-304.

31. Thomas F, Hehemann J-H, Rebuffet E, et al. (2011) Environmental and gut bacteroidetes: the food connection. Front Microbiol 2, 93.

32. Hooper LV, Midtvedt T \& Gordon JI (2002) How hostmicrobial interactions shape the nutrient environment of the mammalian intestine. Annu Rev Nutr 22, 283-307.

33. Xu J, Bjursell MK, Himrod J, et al. (2003) A genomic view of the human-Bacteroides thetaiotaomicron symbiosis. Science 299, 2074-2076.

34. El Kaoutari A, Armougom F, Gordon JI, et al. (2013) The abundance and variety of carbohydrate-active enzymes in the human gut microbiota. Nat Rev Microbiol 11, 497-504.

35. Murphy E, Cotter P, Healy S, et al. (2010) Composition and energy harvesting capacity of the gut microbiota: relationship to diet, obesity and time in mouse models. Gut 59, 1635-1642. 